\title{
Dysmenorrhea Revealing a Pseudo-Horned Uterus at Ouakam Military Hospital in Senegal
}

\author{
Niang $M_{M}^{*}{ }^{*}$, Gaye YFO, Lemine AOM, Diarra CAT, Diouf AA and Cisse CT
}

Obstetrics and Gynecology, Hospital Social Hygiene Institute, Dakar, Senegal

${ }^{*}$ Corresponding author: Mouhamadou Mansour Niang, Obstetrics and Gynecology, Hospital Social Hygiene Institute, Dakar, Senegal, Tel: +221777080007; E-mail: mansniang@hotmail.com

Received date: 30 July, 2016; Accepted date: 30 August, 2016; Published date: 31 August, 2016

Citation: Niang MM, Gaye YFO, Lemine AOM, et al. Dysmenorrhea revealing a pseudo-horned uterus at Ouakam Military Hospital in Senegal, Gynecol Obstet Case Rep. 2016, 2:2.

\section{Abstract}

The pseudo-unicornous uterus also called uterus bicornous unicervical with unequal horns is a rare uterine malformation with a frequency estimated between 2.4 and $13 \%$ of uterine malformations. It is often asymptomatic but the form with functional rudimentary horn, which does not communicate with the uterine cavity, is quite symptomatic and cause primary dysmenorrhea. The diagnosis is made by $3 \mathrm{D}$ ultrasound or magnetic resonance imaging (MRI) and the treatment is surgical.

\section{Key words:}

Pseudo-unicornous uterus; Dysmenorrhea; Magnetic resonance imaging; Laparoscopy

\section{Introduction}

The pseudo-unicornous uterus also called uterus bicornous unicervical with unequal horns is a rare uterine malformation. It is characterized by the existence of a rudimentary horn which can be functional or not, communicating or not with the uterine cavity. This abnormality is due to a melting failure of the Müllerian ducts leading to an incomplete unilateral aplasia.

Its frequency, still poorly known, is estimated between 2.4 and $13 \%$ of uterine malformations [1]. Usually asymptomatic, the pseudo-unicornous uterus can be revealed by pelvic pain, dysmenorrhea or dyspareunia [2]. The non-communicating and functional form, Ilb class of the American Society of Reproductive Medicine (ASRM), is the most common and most symptomatic [1]. The diagnosis is made by $3 D$ ultrasound or magnetic resonance imaging and the treatment is surgical $[1,3]$. Through this case diagnosed and managed at Ouakam Military Hospital in Senegal, we remember the epidemiological, diagnostic and therapeutic aspects of the pseudo-unicornous uterus.

\section{Observation}

Mrs. F., 27 years old, 0 gravida, married since 14 months, consult for cyclic pelvic pain. She reported episodes of intractable dysmenorrhea. Her cycle was regular with menses during 4-5 days. The clinical examination was normal. The 2D ultrasound and the hysterosalpingographia has revealed a uterus unicervical with 2 horns (Figure 1).

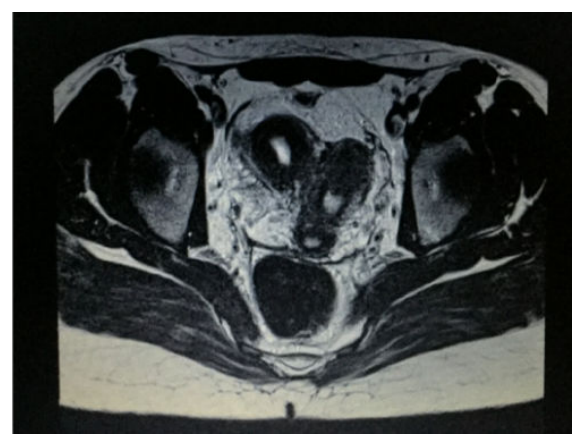

Figure 1 Pseudo-unicornous uterus with rudimentary and functional horn (MRI), arrow resembling rudimentary horn

MRI confirmed the pseudo-unicornous unicervical uterus without communication or renal ectopy or malformation associated (Figure 2).

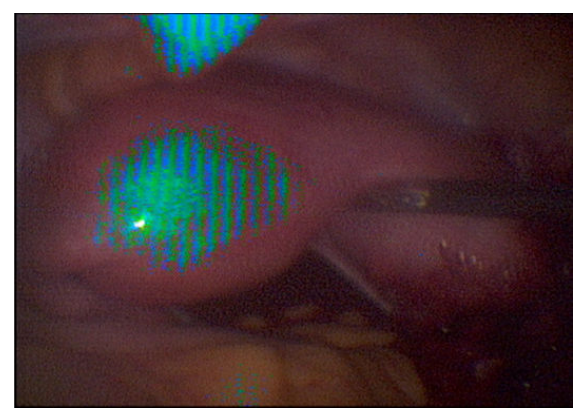

Figure $\mathbf{2}$ Laparoscopic view of a right rudimentary horn, arrow resembling right rudimentary horn 
Laparoscopy revealed a right rudimentary horn about $6 \mathrm{~cm}$ of length with a rudimentary ipsilateral uterine tube and endometriosis nodules on the right ovary. This malformation was classified as IIb of the ASRM.

A right salpingectomy and an excision of the rudimentary horn were performed by laparoscopy. There were no postoperative complications and the patient left the hospital $48 \mathrm{~h}$ after the surgery. The pathological examination of the surgical specimen revealed an adenomyosis.

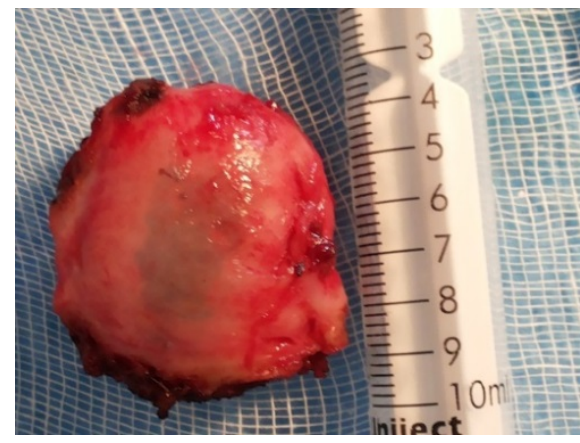

Figure 3 Surgical specimen (right rudimentary horn)

The patient had her period three weeks after surgery without dysmenorrhea. Two months later we have done a second look laparoscopy and there were some adhesions that were cut and the left tube was normal (Figure 4).

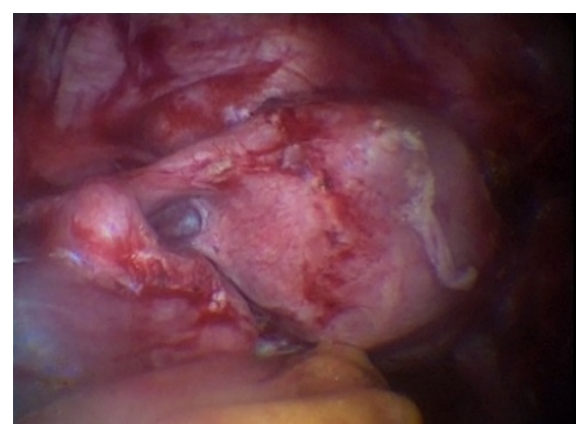

Figure 4 Second look laparoscopic view, arrow showing hemi-uterus with normal tube

\section{Discussion}

The pseudo-unicornous uterus with a rudimentary horn is a rare malformation. The rudimentary horn does not communicate with the uterine cavity in $83 \%$ of cases [4]. It is reported in the literature that this rudimentary horn is usually situated on the right side with a frequency varying between 62 and $80 \%$ according to the authors [5]. For our patient, we have done the same observation. That was a pseudo-unicornous uterus with a right rudimentary horn. Zapardiel and colleagues as well as Borah and colleagues also have reported that observation $[4,6]$.
The rudimentary horn can be contained with an endometrial mucosa, giving it a functional character. It is often asymptomatic, discovered incidentally or during an obstetric complication [4]. However, the form with functional rudimentary horn, which does not communicate with the uterine cavity, is quite symptomatic because of the retention of menses. It causes dysmenorrhea, endometriosis by retrograde menstruation and chronic pelvic pain. This abnormality is associated with endometriosis in more than $40 \%$ [7].

In our patient, the dysmenorrhea revealed the uterine malformation, as in the work of Agarwal and colleagues, Borah and colleagues and Akdemir and colleagues $[1,4,7]$.

Nowadays, MRI and 3D ultrasound are the best diagnostic tools used for the pseudo-unicornous uterus. Zapardiel and colleagues had used the two techniques to diagnose their cases of pseudo-unicornous uterus [6]. In the opposite, in the observation of Agarwal and colleagues the diagnosis was confirmed by MRI [7]. For our patient, we also performed an MRI because 3D ultrasound was not available in our hospital at that time. This exam is now preferred to MRI if the practician is experienced and efficient. Kim and colleagues had shown the superiority of 3D ultrasound comparing to MRI in the diagnosis of pseudo-unicornous uterus with rudimentary horn [3].

These two exams confirm the diagnosis of pseudounicornous uterus and specify if there is or not an association with urinary malformations. The ipsilateral renal agenesis is the most common. It was reported by Agarwal and colleagues and Borah and colleagues in their observations [4,7]. MRI performed in our patient hadn't revealed renal malformation or ectopy.

Laparoscopy performed for the treatment confirmed the diagnosis and revealed endometriosis nodules at the ipsilateral ovary and a rudimentary uterine tube. The course of this situation could be an endometrioma as shown by the work of Agarwal and colleagues and Borah and colleagues [4,7]. An endometriosis cyst on the ipsilateral ovary complicating a pseudo-unicornous uterus with functional non-communicating rudimentary horn was reported in their observations.

Laparoscopic resection has the advantage of reducing the hospital stay period and being more esthetic.

\section{Conclusion}

Primary dysmenorrhea is a frequent reason for consultation. It must impose a further investigation like 3D ultrasound and $\mathrm{MRI}$ in search of an organic cause. Early diagnosis and radical treatment by laparoscopic resection of functional noncommunicating rudimentary horn prevent some serious complications like endometriosis.

\section{References}

1. Akdemir A, Ergenoglu AM, Yeniel AO, Sendag F, Karadadas $\mathbf{N}$ (2014) Coring-type laparoscopic resection of a cavitated noncommunicating rudimentary horn under hysteroscopic assistance. J Obstet Gynaecol Res 40: 1950-1954. 
2. Atmaca R, Germen AT, Burak F, Kafkasli A (2005) Acute Abdomen in a Case With Non-communicating Rudimentary Horn and Unicornuate Uterus. JSLS 9: 235-237.

3. Kim T-H, Lee H-H, Jeon D-S, Park J (2013) Laparoscopic resection of the rudimentary horn of a unicornuate uterus diagnosed by three-dimensional computed tomography. Med Case Stud 4: 9-12.

4. Borah T, Das A, Panda S, Singh S (2010) A case of unilateral dysmenorrhea. J Hum Reprod Sci 9: 158-9.
5. Fedele L, Bianchi S, Zanconato G (2005) Laparoscopic removal of the cavitated non-communicating rudimentary uterine horn: surgical aspects in 10 cases. Fertil Steril 83: 432-436.

6. Zapardiel I, Alvarez P, Perez-Medina T, Bajo-Arenas JM (2010) Laparosopic management of a cavitated non-communicating rudimentary uterine horn of a unicornuate uterus: a case report. J Med Case Rep 4: 1-3.

7. Agarwal M, Das A, Singh A (2011) Dysmenorrhea due to a rare mullerian anomaly. Niger J Clin Pract 14: 377-379. 